
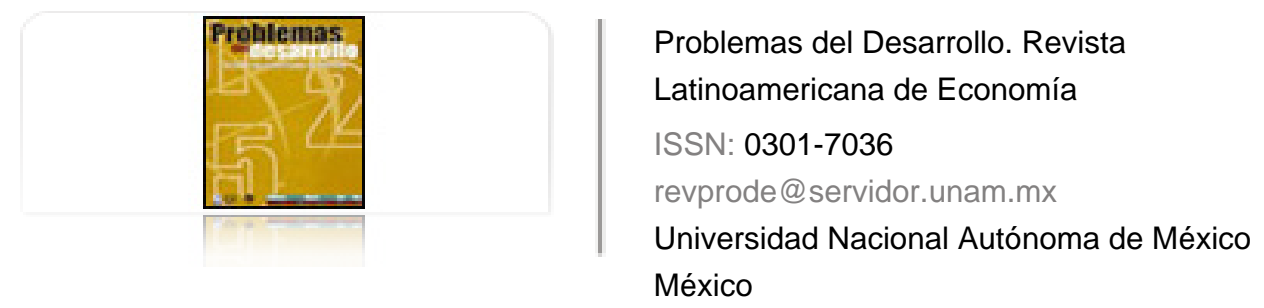

Rosas Rojas, Eduardo

INTERVENCIÓN ESTERILIZADA EN LOS MERCADOS DE CAMBIOS DE AMÉRICA LATINA: BRASIL, CHILE Y MÉXICO

Problemas del Desarrollo. Revista Latinoamericana de Economía, vol. 42, núm. 167, octubrediciembre, 2011, pp. 97-122

Universidad Nacional Autónoma de México

Distrito Federal, México

Disponible en: http://www.redalyc.org/articulo.oa?id=11820101005

- Cómo citar el artículo

- Número completo

- Más información del artículo

Página de la revista en redalyc.org

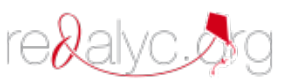

Sistema de Información Científica

Red de Revistas Científicas de América Latina, el Caribe, España y Portugal Proyecto académico sin fines de lucro, desarrollado bajo la iniciativa de acceso abierto 
Revista Problemas del Desarrollo, 167 (42), octubre-diciembre 2011

\title{
INTERVENCIÓN ESTERILIZADA EN LOS MERCADOS DE CAMBIOS DE AMÉRICA LATINA: Brasil, Chile y MÉXICO
}

\author{
Eduardo Rosas Rojas* \\ Fecha de recepción: 6 de enero de 2011. Fecha de aceptación: 13 de junio de 2011.
}

\section{RESUMEN}

Se analiza la pertinencia del modelo macroeconómico convencional en que se apoya el esquema de metas de inflación y se examina su inviabilidad cuando se rechaza la hipótesis de paridad de tasas de interés, lo cual permite utilizar la intervención esterilizada en el mercado cambiario como un instrumento de política monetaria. El objetivo de este trabajo es evaluar por métodos econométricos, si efectivamente tres de los países de América Latina (Brasil, Chile y México) que se autodenominan flotadores, pues sus bancos centrales adoptan esquemas de metas de inflación, y en donde se observa un traspaso magnificado del tipo de cambio a la inflación, utilizan la intervención esterilizada en el mercado cambiario para regular el tipo de cambio.

Palabras clave: Intervención esterilizada, tipo de cambio, política monetaria, paridad descubierta de tasas de interés, miedo a flotar.

\section{STERILIZED INTERVENTION IN LATIN AMERICA'S FOREIGN EXCHANGE MARKETS: Brazil, Chile and Mexico}

\begin{abstract}
We analyze the relevance of the conventional macroeconomic model supporting the inflation target program and examine its non-viability when interest rate parity is rejected, making it possible to apply sterilized intervention in the foreign exchange market as a monetary policy instrument. In this study, we want to use econometric methods to evaluate whether three Latin American countries (Brazil, Chile and Mexico) -which refer to themselves as having floating currencies, as their central banks adopt inflation target programs, and where a magnified pass-through effect is observed from the exchange rate to inflation -effectively use sterilized intervention in the foreign exchange market to regulate their exchange rates.

Key words: Sterilized intervention, exchange rate, monetary policy, exchange rate discovery through interest rates, fear of floating.

* Profesor de Matemáticas y Estadística en la Facultad de Estudios Superiores Acatlán de la unAM. Correo electrónico: eduardor@apolo.acatlan.unam.mx
\end{abstract}


Eduardo Rosas Rojas

\section{L'INTERVENTION STÉRILISÉE SUR LES MARCHÉS DES CHANGES D'AMÉRIQUE LATINE: BRÉSIL, DU CHILI ET DU MEXIQUE \\ Résumé}

Il est procédé ici à l'analyse de la pertinence du modèle macro-économique conventionnel qui s'appuie sur le schéma de ciblage de l'inflation dont on observe qu'il n'est pas viable quand on rejette la condition de parité des taux d'intérêts, ce qui permet d'employer l'intervention stérilisée sur le marché des changes comme un instrument de politique monétaire. L'objectif de ce travail est d'évaluer par des méthodes économétriques, si effectivement trois pays d'Amérique latine (le Brésil, le Chili et le Mexique), qui se qualifient eux-mêmes de flottants du fait que leurs banques centrales adoptent des schémas de ciblage de l'inflation et où on observe une magnifique transition du taux de change à l'inflation, emploient l'intervention stérilisée sur le marché des changes pour réguler le taux de change.

Mots clés : Intervention stérilisée, taux de change, politique monétaire, parité découverte de taux d'intérêt, peur de flotter.

\section{A INTERVENÇÃO ESTERILIZADA NOS MERCADOS DE CÂMBIOS DA AMÉRICA LATINA: BRASIL, CHILE E MÉXICO \\ Resumo}

Analisa-se a pertinência do modelo macroeconômico convencional que se apóia no esquema de metas de inflação e se examina sua inviabilidade quando se rechaça a hipótese de paridade das taxas de juros, o qual permite utilizar a intervenção esterilizada no mercado cambiário como um instrumento de política monetária. O objetivo deste trabalho é avaliar por métodos econométricos, se efetivamente três destes países de América Latina (Brasil, Chile e México) que se auto-dominam praticantes do cambio flutuante, pois seus bancos centrais adotam esquemas de metas de inflação, e onde se observa um transpasso importante do tipo de cambio à inflação, utilizam a intervenção esterilizada no mercado cambiário para regular o tipo de cambio.

Palavras-chave: Intervençáo esterilizada, tipo de cambio, política monetária, paridade descoberta da taxa de juros, medo a flutuar.

沖銷千預在拉丁美洲貨幣市場在巴西，智利和墨西哥

摘要

我們分析了常規的宏觀經濟模型, 支持通貨膨脹目標框架和審視自己 的不切實際時, 拒了利率平價, 這使得作為一種工具的消毒千預外龨 市場使用的相關假說貨幣政策。這項工作的目的是評估的經濟計量方 法，如果確實拉丁美洲（巴西，智利和墨西哥）的三個國家的彩車自 己, 因為他們的中央銀行採用通貨膨脹目標的計劃, 並在有一個放大 傳輸匯率, 通貨膨脹, 利用外匯市場沖銷千預來調節匯率

關鍵詞：沖銷千預，匯率，貨幣政策，利率平價發現，浮動恐懼 


\section{INTRODUCCIÓN}

La mayoría de los bancos centrales de las economías emergentes afirman que el actual régimen cambiario es de libre flotación y que la política monetaria ha sustituido al tipo de cambio como ancla nominal de la economía. Las estadísticas revelan que un gran número de países que permiten la flotación de sus monedas en realidad no la llevan a cabo (Calvo y Reinhart, 2002; Reinhart y Rogoff, 2004; Böfinger y Wollmershäuser, 2001; Hüfner, 2004; etcétera). Este fenómeno económico conocido como "miedo a flotar" está comprobado especialmente en los países que experimentan un elevado traspaso del tipo de cambio a la inflación, y que muestran un compromiso de sus bancos centrales por aplicar esquemas de metas de inflación.

Las investigaciones empíricas realizadas por Böfinger (2001) y Hüfner (2004) demuestran el incumplimiento de la paridad de tasas de interés en el corto y largo plazos. Esta teoría se basa en dos supuestos: $i$ ) que los fundamentales de las economías se reflejan en la variación esperada del tipo de cambio; y ii) que los activos financieros son perfectamente sustituibles. Al cumplirse estos supuestos, las variaciones en los diferenciales de tasas de interés determinan las variaciones del tipo de cambio, de tal forma que la política monetaria se podría conducir con un solo instrumento (Romer, 2000). Sin embargo, la violación del supuesto de paridad de tasas de interés en el modelo en que se sustenta el régimen de metas de inflación permite utilizar dos instrumentos de política monetaria en lugar de sólo uno: $i$ ) las operaciones de mercado abierto en el mercado de dinero para establecer la tasa de interés y ii) la intervención esterilizada en los mercados de cambios, para regular el tipo de cambio.

El objetivo de este trabajo es examinar por métodos econométricos, si efectivamente tres de los países en desarrollo de América Latina (Brasil, Chile y México) que se autodenominan flotadores pues adoptan esquemas de metas de inflación, y en los cuales se observa un traspaso magnificado del tipo de cambio a la inflación, utilizan la intervención esterilizada en el mercado cambiario para regular el tipo de cambio.

El trabajo está compuesto por tres secciones:

En la sección uno se analiza la pertinencia del modelo macroeconómico convencional en que se apoya el esquema de metas de inflación, y se examina su inviabilidad cuando se rechaza la hipótesis de paridad de tasas de interés, lo cual permite utilizar la intervención esterilizada en el mercado cambiario como un instrumento de política monetaria. Se muestra evidencia empírica sobre la existencia del "miedo a flotar" en las economías emergentes de América Latina, y se analizan sus posibles causas. Finalmente, se fundamenta la utilización 
de la flotación administrada como mecanismo para superar el triángulo de inconsistencia.

En la segunda sección se desarrolla un modelo para probar el uso de la intervención esterilizada como instrumento de política, que es una extensión del modelo de Böfinger y Wollmershäuser (2001). Posteriormente esta hipótesis se somete a prueba mediante la estimación de un modelo econométrico de panel no balanceado, en una muestra de 3 países, Brasil, Chile y México, durante el periodo 2002: 1 a 2008: 12 .

En la tercera sección se describen y analizan los resultados obtenidos en el modelo econométrico. Se señalan tanto los beneficios como los costos reales y financieros derivados del uso excesivo de la intervención esterilizada en los mercados de cambios de los países estudiados, así como los riesgos sistémicos asociados.

\section{LA INTERVENCIÓN ESTERILIZADA EN EL MERCADO DE CAMBIOS}

\section{El triángulo de inconsistencia}

Por décadas se ha sostenido que un sistema económico no puede funcionar bajo un marco de libre movilidad de capitales y control simultáneo sobre el tipo de cambio y la tasa de interés. Lo anterior se define como el triángulo de inconsistencia o la triada imposible (Böfinger y Wollmerschäuser, 2001).

En condiciones de exceso de demanda de moneda extranjera en el mercado cambiario, aun los bancos centrales poderosos tienen una capacidad de intervención en el mercado de cambios, limitada por su disponibilidad de reservas, y en consecuencia, el triángulo de inconsistencia es generalmente válido.

Sin embargo, cuando el banco central establece una meta para el tipo de cambio y se presenta un exceso de oferta de moneda internacional el triángulo de inconsistencia queda invalidado (Böfinger y Wollmershäuser, 2001; Frenkel, 2007). ${ }^{1}$ En esta situación, el banco central tiene la facultad para determinar simultáneamente el tipo de cambio y la tasa de interés.

1 De acuerdo con Frenkel (2007) el exceso de oferta de moneda internacional al tipo de cambio meta del Banco Central es lo que invalida el trilema y faculta al Banco Central para determinar el tipo de cambio y la tasa de interés. 


\section{Régimen de metas de inflación (MI)}

El modelo macroeconómico en el cual se sustenta el régimen de metas de inflación (Svensson, 2000; Ball, 1999) se basa en la teoría de la nueva síntesis neoclásica, que se resume en cuatro ecuaciones que explican el comportamiento del producto, los precios, la tasa de interés del banco central, y el tipo de cambio.

$\begin{array}{ll}\mathrm{Y}=\mathrm{f}(\mathrm{r}, \mathrm{e}) & \text { Curva Is } \\ \mathrm{P}=\mathrm{f}(\mathrm{Y}, \mathrm{e}) & \text { Curva de Phillips } \\ \mathrm{i}=\mathrm{f}\left(\mathrm{Y}, \mathrm{P}-\mathrm{P}^{*}\right)=\mathrm{r}+\mathrm{P} & \text { Regla de Taylor o función de costo social } \\ \mathrm{e}=\mathrm{f}\left(\mathrm{i}, \mathrm{i}^{*}\right) & \text { Paridad de tasas de interés }\end{array}$

Donde $\mathrm{Y}$ es el producto real, $\mathrm{r}$ la tasa de interés real, $\mathrm{P}$ es la tasa de inflación observada, $\mathrm{P}^{*}$ es la meta de inflación establecida, e es el tipo de cambio, i es la tasa de interés nominal que controla el banco central, e $\mathrm{i}^{*}$ es la tasa de interés externa.

El modelo teórico de metas de inflación asume que el tipo de cambio se determina por libre flotación, y que la elasticidad de los flujos de capital a corto plazo respecto a las tasas de rendimiento es muy elevada, por lo que sería inútil que los bancos centrales trataran de intervenir en el mercado cambiario aumentando la oferta o la demanda de divisas, ya que cualquier acción tendría efectos monetarios que causarían una reacción compensatoria en los flujos de capital. Dicho en otras palabras, este modelo asume que se cumple la condición de paridad descubierta de tasas de interés.

Bajo la hipótesis de que se cumple la paridad descubierta de tasas de interés, la intervención esterilizada no es reconocida como un instrumento en los modelos de metas de inflación, que suponen mercados eficientes. Esto se debe a que el tipo de cambio se asocia exclusivamente a la tasa de interés, y el signo de dicha asociación solamente cambia dependiendo del horizonte de tiempo que se esté considerando. Es decir, es negativo en el corto plazo, tal como lo concibe el modelo Mundell-Fleming, y positivo en el largo plazo, lo cual se explica con la paradoja de Fisher dentro del modelo del Enfoque Monetario de Balanza de Pagos (EMBAP).

Así, el instrumento por excelencia en el régimen de metas de inflación es la tasa de interés, en algunas ocasiones complementada con el seguimiento de los agregados monetarios. Esta instrumentación de la política monetaria también depende del grado de desarrollo del mercado financiero y de la capacidad de fijar tasas interbancarias. Adicionalmente, se debe contar con un banco central independiente que permita establecer efectivamente la política monetaria. 
Eduardo Rosas Rojas

\section{Incumplimiento de la Paridad de Tasas de Interés}

Una elevada elasticidad de los flujos de capital externo respecto a la tasa de interés puede ser razonable en un país con moneda dura, es decir, moneda de reserva, pero es utópico en países en desarrollo con mercados financieros estrechos y delgados y déficit crónico en la balanza de pagos, que debilitan sus monedas (Toporowski, 2005). En las economías en desarrollo vemos que la hipótesis de paridad de tasas de interés descubierta no se cumple.

El incumplimiento de la paridad de tasas de interés ha tenido diversas explicaciones. Entre las más importantes resalta la de Hüfner (2004), establece que los inversionistas con horizontes de planeación a corto plazo piden prestado en una moneda para invertir en valores gubernamentales en otra moneda (carrytrade), y al explotar los diferenciales de rendimiento contribuyen a la apreciación de las monedas que pagan mayores tasas de interés.

En el largo plazo, la paridad descubierta de tasas de interés tampoco se ha visto apoyada por la evidencia empírica. Hüfner (2004) puso a prueba la validez de la teoría de la paridad de tasas de interés descubierta, como la contempla el enfoque monetario de la balanza de pagos (i.e. como la paradoja de Fisher), en cinco países con metas de inflación. Para este fin, relacionó las variaciones corrientes en los tipos de cambio con los diferenciales rezagados de las tasas de interés domésticas respecto a la tasa de interés de depósitos en dólares, bajo la hipótesis nula de que las monedas que pagaran mayores tasas de interés serían las que posteriormente se devaluarían frente al dólar. En los cinco casos, el parámetro del diferencial de tasas de interés resultó significativamente negativo, con lo cual se rechaza que la depreciación del tipo de cambio lleve a la nivelación de las ganancias de las inversiones entre los países, evidenciando que los inversionistas internacionales explotan a corto plazo los diferenciales (mediante el carry trade).

El enigma de la prima adelantada (forward premium puzzle) explica la apreciación de las monedas que pagan mayores intereses y está relacionado al incum-

plimiento de la paridad descubierta de tasas de interés. El enigma comprueba que la prima adelantada usualmente apunta en la dirección equivocada (apreciación) para el movimiento ex post en el tipo de cambio al contado, lo cual contradice el supuesto de expectativas racionales. Los resultados soportan lo establecido por Flood y Rose (2001), quienes establecen que si bien la paridad de tasas de interés descubierta es un tema clásico de las finanzas internacionales, y un pilar fundamental de la mayoría de los modelos teóricos, también es cierto que es un triste fracaso empírico.

La violación del supuesto de paridad de tasas de interés en el modelo teórico en que se sustenta el régimen de metas de inflación, permite usar dos 
instrumentos de política monetaria en lugar de sólo uno: $i$ ) las operaciones de mercado abierto para establecer la tasa de interés, e ii) la intervención esterilizada para regular el tipo de cambio.

\section{La intervención esterilizada en el mercado cambiario como un instrumento de política monetaria}

Cuando se esteriliza un impacto monetario mediante la intervención que lleva a cabo la autoridad monetaria se dice que dicho mecanismo constituye un instrumento de política independiente de la tasa de interés.

La idea de que la intervención directa de los bancos centrales en los mercados cambiarios es ineficaz en los países en desarrollo ha cambiado significativamente en las últimas décadas. A ello ha contribuido un estudio extenso de Canales-Kriljenko (2003), basado en los resultados de la encuesta del FMI de 2001 sobre la organización de los mercados cambiarios. Uno de los principales resultados en este documento es que los bancos centrales en muchos mercados emergentes pueden ser capaces de llevar a cabo la intervención cambiaria con más eficacia que los bancos centrales de los países emisores de las principales monedas internacionales.

Sin embargo, este instrumento no es reconocido en los modelos de metas de inflación, sustentados en las teorías monetarias ortodoxas del tipo de cambio que suponen la existencia de mercados eficientes y el cumplimiento de la paridad descubierta de tasas de interés.

\section{El "miedo a flotar" en las economías emergentes}

Las investigaciones recientes indican que un gran número de países que declaran estar flotando sus monedas, en realidad no lo están llevando a cabo. A este fenómeno se le conoce como el "miedo a la flotación" (Fear of Floating), es decir, el uso de la política monetaria para estabilizar el tipo de cambio a costa de volatilidad en la tasa de interés dentro de un régimen de flotación (Ibarra, 2007).

Böfinger y Wollmershäuser (2001) encontraron que de 66 regímenes reportados al FMI como de tipo de cambio flexible, sólo 4 toleran una flotación pura; 7 corresponden a flotación independiente; y 55 practican una flotación administrada. 
Eduardo Rosas Rojas

\section{Régimen de tipo de cambio de flotación administrada}

La flotación administrada (managed floating) implica que la dirección del tipo de cambio está determinada por el banco central (o el gobierno); además no hay una ruta "preanunciada" para el tipo de cambio. Este régimen proporciona una solución óptima para lograr el equilibrio interno y externo, aunque se necesita de un dispositivo institucional adicional. La solución más obvia son las metas de inflación que proveen un ancla para las expectativas mediante un anuncio público de las tasas de inflación que el banco central intenta lograr (Böfinger y Wollmershäuser, 2001). No hay nada que impida una combinación de flotación administrada y metas de inflación. Mientras el equilibrio interno es definido como un nivel de producción que implica pleno empleo, el equilibrio externo es definido como una situación en la que las reservas de divisas del banco central permanecen constantes.

Böfinger y Wollmershäuser (2001) afirman que se puede superar el triángulo de inconsistencia a través de la flotación administrada. Estos autores proponen como solución óptima que el nivel de tasa de interés y el sendero del tipo de cambio estén determinados simultáneamente, que la intervención esterilizada tenga cabida dentro del esquema de política monetaria. El marco teórico desarrollado en esta sección, permite afirmar que dado un exceso de oferta de moneda internacional al tipo de cambio meta del banco central, la paridad de tasas de interés puede incumplirse.

\section{MODELO ECONOMÉTRICO PARA MEDIR EL GRADO DE INTERVENCIÓN ESTERILIZADA EN PAÍSES DE AMÉRICA LATINA PARA EL PERIODO 2002-2008}

La forma de verificar si la autoridad monetaria efectivamente ha abandonado el ancla cambiaria y aplica rigurosamente el esquema de metas de inflación para anclar las expectativas del público es midiendo por métodos econométricos el grado de intervención esterilizada del banco central en el mercado de cambios (Mántey, 2009).

Se elaborará un modelo econométrico de datos de panel no balanceado. Böfinger y Wollmershäuser (2001) proponen un modelo para medir el grado en que la autoridad monetaria recurre a la intervención esterilizada en el mercado cambiario, en el cual relacionan la variación de los activos internos del banco central (NDA) con la variación de sus activos externos (NFA). El modelo se expresa de la siguiente manera:

$$
\Delta \mathrm{NDA}_{\mathrm{t}}=\mathrm{a}+\mathrm{b} \Delta \mathrm{NFA}_{\mathrm{t}}+\mathrm{c} \Delta \mathrm{NDA}_{\mathrm{t}-1}+\mathrm{u}
$$


Bajo una completa esterilización, el coeficiente "b" del cambio en las reservas netas extranjeras $\triangle N F A_{t}$ se espera que sea -1 . Los valores rezagados de ( $\triangle \mathrm{NDA}$ ) son incluidos para capturar otros efectos de la política de esterilización del banco central.

\section{Planteamiento del modelo teórico}

A fin de medir el grado de intervención esterilizada que practican los bancos centrales de Brasil, Chile y México, se estima un modelo similar al propuesto por Böfinger y Wollmershäuser (2001), ${ }^{2}$ pero en vez de incluir el término rezagado de los activos domésticos netos se incorporan otras variables que influyen en la capacidad de esterilización, que contribuyen a generar una oferta excedente de divisas. Se consideran explícitamente tres elementos adicionales que influyen en la esterilización:

- El aumento en la demanda de base monetaria (м) Frenkel (2007). La intervención esterilizada es una política instrumentada en dos pasos. En el primer paso, la intervención del banco central en el mercado cambiario genera una expansión de la base monetaria. La situación resultante mostraría un stock mayor de base monetaria, el stock inalterado de activos domésticos y una tasa de interés menor que la inicial. En el segundo paso, la esterilización completa compensa totalmente el cambio en el portafolio privado que tuvo lugar en el primer paso. El banco central absorbe el incremento de la base monetaria y coloca un monto de activos domésticos equivalente al exceso de demanda de activos domésticos inicial (el exceso de oferta de divisas) reponiendo la tasa de interés doméstica a su nivel previo (Böfinger and Wollmershäuser, 2003).

- Un indicador de oferta excedente de divisas, el cual se representará por el diferencial de tasa de interés interna y la tasa de interés externa más el riesgo país.

- El patrimonio neto del banco central (Nw), incluye los superávits acumulados por tenencias de activos netos internos y extranjeros (Sarno, 2000).

2 De acuerdo con Böfinger y Wollmershäuser (2001), la ecuación de esterilización es:

$$
\Delta \mathrm{NDA}_{\mathrm{t}}=\beta_{1} \Delta \mathrm{NFA}_{\mathrm{t}}+\beta_{2} \Delta \mathrm{NDA}_{\mathrm{t}-1}+\mathrm{Ut}
$$

Bajo una completa esterilización el coeficiente $\beta 1$ del cambio en las reservas netas extranjeras $\triangle N F A_{t}$ se espera que sea de -1, para el crédito neto doméstico (activos domésticos netos) $\triangle \mathrm{NDA}_{\mathrm{t}}$. 
El modelo está representado por medio de factores influyentes, conformados por variables representativas o variables proxy, conforme a la especificación del siguiente modelo:

$\Delta$ AIMIDL $_{\mathrm{it}}=\mathrm{a}+\mathrm{b} \Delta \mathrm{BCCNTDL}_{\mathrm{it}}+\mathrm{c} \Delta \mathrm{MDL}_{\mathrm{it}}+\mathrm{d} \Delta \mathrm{NW}_{\mathrm{it}}+\mathrm{e} \Delta \mathrm{TMIMLRP}_{\mathrm{it}}+\mathrm{e}_{\mathrm{it}}$

Bajo la hipótesis planteada se espera a priori que $-1<\mathrm{b}<0$; mientras que c,d,e $>0$.

Donde:

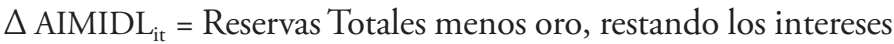

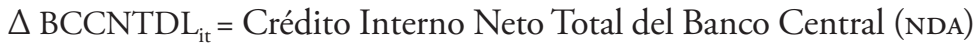

$\triangle \mathrm{MDL}_{\mathrm{it}}=$ Base Monetaria (considera los pasivos de reserva de los bancos comerciales y de otros sectores)

$\Delta \mathrm{NW}_{\mathrm{it}}=$ El Patrimonio Neto de las autoridades monetarias

$\triangle$ TMIMLRP $_{\text {it }}=$ Tasa del Mercado Monetario menos (Tasa Libor + EMBI)

$\mathrm{e}_{\mathrm{it}}=$ Término de perturbación de la i-ésima unidad transversal (país) y $t$ el tiempo $t$ (mes).

\section{Descripción de las variables usadas}

Siguiendo la metodología de Böfinger y Wollmershäuser (2001), se elaboraron variables proxy para representar los incrementos en los activos externos netos; para ello se calcularon los incrementos en las reservas menos el oro descontando los intereses devengados (AIMIDL), utilizando la tasa de rentabilidad de los bonos del gobierno de los Eevu o bien la tasa Libor.

Las variaciones en los activos internos netos del banco central (BCCNTDL) se calcularon mediante un desglose del crédito interno otorgado por el instituto emisor al gobierno, a sus intermediarios financieros internos y a otros sectores, una vez que se restaron los pasivos con cada uno de ellos.

Para capturar los efectos de otros factores influyentes en la capacidad de esterilización del banco central, se agregaron al modelo las siguientes variables: 1) los pasivos de reserva a los bancos comerciales y a otros sectores; 2) el diferencial entre las tasas de interés del mercado interbancario y la tasa de préstamos más el riesgo país, y 3) el patrimonio neto del banco central con la finalidad de capturar los superávits acumulados por tenencias de activos netos internos y extranjeros. 


\section{Metodología econométrica}

La metodología que se utilizó para estimar el modelo se denomina modelo econométrico de datos de panel no balanceado, el cual incluye una muestra de algunos países latinoamericanos (Brasil, Chile y México) para un periodo determinado (2002: 1 a 2008: 12), esto es, combina ambos tipos de datos (dimensión temporal y estructural).

El principal objetivo de aplicar y estudiar los datos de panel, es capturar la heterogeneidad no observable: $i$ ) los efectos individuales específicos y ii) los efectos temporales; entre agentes económicos, dado que esta heterogeneidad no se puede detectar sólo con estudios de series temporales, ni tampoco con los de corte transversal (Burdisso, 1997).

La técnica de datos de panel permite disponer de un mayor número de observaciones incrementando los grados de libertad y reduciendo la colinealidad entre las variables explicativas y, en última instancia, mejorando la eficiencia de las estimaciones econométricas (Hsiao, 1986).

\section{Análisis de integración de las series}

Utilizar series de tiempo no estacionarias en el análisis de regresión presenta serias dificultades, en la medida en que las pruebas de hipótesis convencionales, los intervalos de confianza y las predicciones del futuro a partir del modelo no van a ser confiables, ya que estará presente la regresión espuria en la terminología de Granger y Newbold (1974). ${ }^{3}$ El problema con estas regresiones es que tienden a admitirse como buenas, relaciones económicas que, en realidad, sólo se deben a aspectos casuales.

A finales de la década de los setenta y comienzos de los ochenta, Dickey y Fuller $(1979,1981)$ propusieron pruebas estadísticas formales para determinar si una serie de tiempo es estacionaria en diferencia, o si es estacionaria alrededor de una tendencia determinística. Este tipo de pruebas se conocen en la literatura como "pruebas de raíz unitaria". Las pruebas de raíz unitaria para datos de panel

3 El fenómeno de la regresión espuria fue descubierto por primera vez por G. U. Yule, "Why do we Sometimes get Nonsense Correlations Between Time Series? A Study in Sampling and the Nature of Time Series", en Journal of the Royal Statistic Society, vol. 89, 1926, pp. 1-64.

De acuerdo con Granger y Newbold (1974), una $\mathrm{R}^{2}>$ Durbin Watson, es una Buena regla práctica para sospechar que la regresión estimada es espuria. 
que contienen la mayoría de los paquetes estadísticos son: Levin y Lin (1992) (LL), posteriormente Im, Pesaran y Shin (2003) (IPS), la prueba tipo Fisher usando ADF y PP. Todas las variables utilizadas resultaron ser estacionarias en primeras diferencias, o dicho de otra forma, tienen un grado de integración uno.

\section{Prueba de causalidad de Granger}

La prueba de causalidad de Granger es ampliamente conocida en la práctica econométrica ya que permite identificar de forma rápida relaciones de causalidad entre las variables explicativas y la variable a explicar. El objetivo de la prueba es determinar si una variable BCCNTDL causa a otra variable AIMIDL o viceversa. Los resultados muestran que la variable BCCNTDL es causal en términos de Granger de la variable AIMIDL.

Con base en los resultados podemos determinar que en países con regímenes cambiarios de flotación controlada, el tamaño de las reservas de activos internacionales tiene una relación directa con la capacidad de los bancos centrales para intervenir de una manera discrecional en los mercados cambiarios.

\section{IMPLICACIONES DE LA INTERVENCIÓN ESTERILIZADA}

El modelo teórico dominante en el que se apoya el régimen de metas de inflación no describe de manera adecuada el mecanismo de transmisión de la política monetaria en los países latinoamericanos. El modelo econométrico revela que la intervención esterilizada en el mercado de cambios es un instrumento de suma importancia para la política monetaria, el cual es utilizado por los bancos centrales para regular el tipo de cambio, y de esta manera poder cumplir con sus objetivos de metas de inflación.

\section{Diferencial de tasas de interés}

El modelo econométrico para la acumulación de reservas muestra que la elevación de la tasa de interés interbancaria con respecto a la tasa Libor, tiene un coeficiente positivo, indicando que la presión de la autoridad monetaria induce a los bancos comerciales a fondearse en el extranjero, y a cambiar sus disponibilidades en moneda extranjera a moneda nacional, aumentando de esta manera las reservas internacionales del banco central. 
A pesar de lo establecido anteriormente, la condición de paridad de tasas de interés sigue sin cumplirse pues como se planteó anteriormente, los operadores de divisas en el mercado cambiario tienen una estrategia de corto plazo y sólo mantienen posiciones de divisas intra-día, por lo que el diferencial de tasas de interés no influye en sus decisiones de negociación, ni afecta el comportamiento del tipo de cambio.

Esta forma en que se implementa la política monetaria y cambiaria en algunas economías latinoamericanas, especialmente el caso de México en las últimas tres décadas ha derivado en:

1. la apreciación del tipo de cambio con el fin de abatir la inflación, ha aumentado las importaciones y reducido las exportaciones, deteriorando la balanza comercial y restringiendo el crecimiento económico;

2. el lento crecimiento y el mantenimiento de tasas de interés competitivas (i.e. que compensa el riesgo país) han provocado desequilibrios fiscales;

3. riesgos sistémicos, por la aparición de los déficit gemelos, que inducen reversiones violentas de capital externo de corto plazo;

4. desequilibrios en el sector financiero por el fondeo de moneda extranjera de los bancos.

La estabilización y apreciación del tipo de cambio favorecen al capital financiero internacional y contribuyen al proceso de extranjerización de las economías, que han profundizado la dependencia externa tecnológica y científica de los países en vías de desarrollo.

\section{Consecuencias de una acumulación de reservas prolongada}

Altos niveles de reservas juegan un rol importante como mecanismo preventivo cuando los fundamentales se desvían de su trayectoria. En un aporte reciente, Li y Rajan (2005) han formalizado la idea de que, en general, estos activos pueden compensar parcialmente una configuración endeble de las variables macroeconómicas. Sin embargo, en un punto determinado de debilidad de los fundamentales, ningún nivel de reservas reducirá las posibilidades de ocurrencia de un ataque especulativo.

La acumulación prolongada de reservas mediante la intervención esterilizada para cumplir las metas de inflación genera:

1. los costos cuasifiscales de la intervención;

2. futuros desequilibrios monetarios. 
Por otra parte, la acumulación prolongada de reservas es contraria a la transparencia en la conducción de la política económica que supuestamente caracteriza al régimen de metas de inflación.

\section{Incremento de la deuda pública interna como resultado de la política de esterilización}

Frenkel (2008) establece que para evitar caer en un déficit cuasifiscal y mantener un grado considerable de autonomía monetaria se debe cumplir la condición de sostenibilidad de la política de esterilización; esto significa que dicha política sólo es sostenible si la relación entre pasivos totales del banco central (acervo de base monetaria y acervo de pasivos remunerados) y el valor en moneda local de las reservas internacionales no se incrementa. Por tal motivo se incluyó en el modelo econométrico una variable que muestra que la acumulación de reservas está influida en un -0.7181459 con respecto al crédito neto total del banco central y otra variable que representó el señoreaje (base monetaria) con una influencia del 0.2866068 .

La intervención esterilizada para apreciar el tipo de cambio afecta la inversión productiva y el crecimiento económico del país, ya que compromete los ingresos públicos en pagar los intereses de una creciente deuda interna y obstaculiza otros objetivos de la política fiscal.

Aunado a lo anterior se debe reconocer que el elevado y persistente traspaso de variaciones del tipo de cambio a precios que muestran las economías en desarrollo, evidencia la inaplicabilidad de las teorías convencionales para explicar el proceso de formación de precios en ellas.

\section{Traspaso del tipo de cambio a la inflación}

Las teorías heterodoxas de oligopolio ${ }^{4}$ y la teoría estructuralista de la inflación coinciden al postular la exogeneidad de los precios y las utilidades, y la

\footnotetext{
$4 \quad$ Kalecki (1954) sostiene que el nivel de los precios se explica por la estructura de costos de las empresas y el margen de utilidades máximo que pueden obtener, dado el grado de monopolio. El grado de monopolio se determina por el proceso de concentración industrial, que permite la aparición y expansión de grandes empresas, y su capacidad de colusión (tácita o explícita) con otras firmas, para mantener los precios por arriba de sus costos de producción.
} 
endogeneidad de los salarios reales; ambas contemplan el proceso inflacionario como producto de una pugna distributiva, es decir, como un problema de utilidades y no solamente como un fenómeno de presión de demanda o presión de costos (Hernández, 2009).

La concepción de la inflación como un problema distributivo puede explicar el traspaso desproporcionadamente alto del tipo de cambio a la inflación, que muestran las economías latinoamericanas, por un canal de transmisión alterno determinado por las utilidades que no está contemplado en los canales convencionales (i.e. directo, de costos y de demanda). ${ }^{5}$

La disminución del traspaso magnificado del tipo de cambio a la inflación es un pre-requisito para que posteriormente se pueda aplicar un régimen macroeconómico alterno, cuyo componente cambiario estimule altas tasas de crecimiento del producto interno bruto y del empleo pero sin generar inflación.

\section{CONCLUSIONES}

El análisis econométrico realizado para examinar la política monetaria en América Latina nos lleva a concluir que el modelo teórico dominante en el que se apoya el régimen de metas de inflación no describe fielmente su mecanismo de transmisión, y que los bancos centrales de estos países no han abandonado el ancla cambiaria.

Los resultados de diversas investigaciones destacan la presencia de un fenómeno económico denominado "miedo a flotar", el cual está propagado particularmente en los países en desarrollo que experimentan un elevado traspaso del tipo de cambio a la inflación. Esto constituye una prueba contundente de que las autoridades monetarias utilizan la intervención esterilizada en los mercados cambiarios como un instrumento de política complementario. Abonando a lo anterior, el modelo econométrico muestra que existe una acumulación prolongada de reservas, contraria a la transparencia en la conducción de la política económica y a la comunicación con el público, factores que en teoría caracterizan al régimen de metas de inflación.

Se comprobó que los países de América Latina (Brasil, Chile y México) que se autodenominan flotadores, utilizan la intervención esterilizada en el mercado

5 Hernández (2009) establece que el mecanismo de transmisión del tipo de cambio a la inflación que explica el traspaso unitario en estas economías opera por el canal de las utilidades de las empresas locales, que racionalmente optan por seguir al líder en lugar de inciar una guerra de precios 
de cambios para regular el tipo de cambio, y de esta manera cumplir con sus objetivos de metas de inflación.

La experiencia señala que el control de la inflación a través de la apreciación del tipo de cambio resta competitividad a la producción doméstica y genera riesgos sistémicos por la aparición de los déficit gemelos, que causan reversiones violentas de capital externo de corto plazo.

La apreciación cambiaria derivada de la intervención esterilizada compromete los ingresos públicos en pagar los intereses de una creciente deuda interna, y obstaculiza otros objetivos de la política fiscal como son la creación de infraestructura y otros estímulos a la inversión productiva.

Por ello es conveniente implementar un régimen cambiario cuyo objetivo sea un tipo de cambio competitivo y estable, que fomente un uso más intensivo de la fuerza de trabajo y mantenga tasas de interés moderadas, con lo cual se limite el servicio de la deuda pública interna y se privilegie una política fiscal de fomento económico.

Sin embargo, para llevar a cabo el ajuste del tipo de cambio nominal es de fundamental importancia considerar el problema de su traspaso magnificado a la inflación, reconociendo que este proceso es producto de una pugna distributiva y de la dolarización de las economías y del no monopolio del banco central en la tenencia de divisas. La reducción del traspaso magnificado es un requisito previo para poder implantar un régimen de tipo de cambio real competitivo y estable que fomente el crecimiento económico equitativo. 


\section{ANEXOS}

\section{ESTIMACIÓN DEL MODELO}

1.1.1 Regresión agrupada (Pooled M. C. O.)

El enfoque más simple para analizar datos de panel es omitir las dimensiones del espacio y el tiempo de los datos agrupados y sólo calcular la regresión MCO usual. Este modelo se expresa como:

$$
Y_{i t}=\alpha+\beta_{1} X_{1 i t}+e_{i t}
$$

Donde $i$ significa la $i$-ésima unidad transversal (países) y $t$ el tiempo $t$ (meses).

\begin{tabular}{lccc}
\multicolumn{4}{l}{ Cuadro 1 Regresión Agrupada (pooled ols) } \\
\hline Fuente & SS & df & MS \\
Modelo & $1.24 \mathrm{E}+09$ & 4 & $3.1 \mathrm{E}+08$ \\
Residuales & 678632688 & 244 & 2781282 \\
Total & $9.18 \mathrm{E}+08$ & 248 & 7734237 \\
\hline
\end{tabular}

\begin{tabular}{lr}
\hline Número de Obs & 249 \\
$\mathrm{~F}(4,244)$ & 111.41 \\
Prob $>\mathrm{F}$ & 0.0000 \\
R-cuadrada & 0.6462 \\
$\begin{array}{l}\text { R-cuadrada } \\
\text { Ajust. }\end{array}$ & 0.6404 \\
Raíz MSE & 1667.7 \\
\hline
\end{tabular}

\begin{tabular}{lrrrrrr}
\hline \multicolumn{1}{c}{ dlaimidl } & Coef. & Error Est. & \multicolumn{1}{c}{$t$} & \multicolumn{1}{c}{$p>$ It } & \multicolumn{2}{c}{ [Int. de Conf. 95\% ] } \\
\hline dl lbccntdl & -0.718146 & 0.0366067 & -19.62 & 0.000 & -0.7902515 & -0.64604 \\
dlmdl & 0.2866068 & 0.0305602 & 9.38 & 0.000 & 0.2264113 & 0.346802 \\
dlnwdl & 0.7441394 & 0.0378301 & 19.67 & 0.000 & 0.6696242 & 0.818655 \\
dltmimlrp & 0.726577 & 0.1956587 & 3.71 & 0.000 & 0.3411814 & 1.11973 \\
cons & 318.697 & 109.4643 & 2.91 & 0.004 & 103.0814 & 534.3126 \\
\hline
\end{tabular}

Fuente: elaboración propia.

La regresión agrupada toma la información como un todo sin discriminar los datos temporales o transversales. Esta estimación es útil para dilucidar patrones preliminares, los signos y las magnitudes de las variables independientes.

Al examinar los datos de la regresión agrupada se puede ver que todos los coeficientes son individual y estadísticamente significativos, vemos que los coeficientes

$\overline{6}$ Las series de tiempo del modelo se pueden localizar en: http://revistas.unam.mx/index.php/pde 
tienen los signos que se esperaban, y que el valor $R^{2}=0.6462$ es razonablemente alto.

\subsubsection{Efectos aleatorios (Random Effects)}

La ecuación (i) supone que el intercepto de la regresión es el mismo para todas las unidades transversales. Sin embargo, es muy probable que necesitemos controlar el carácter "individual" de cada país. El modelo de efectos aleatorios permite suponer que cada unidad transversal tiene un intercepto diferente. Este modelo se expresa como:

$$
Y_{i t}=\alpha_{i}+\beta_{1} X_{1 i t}+e_{i t}
$$

Donde $\alpha_{i}=\alpha+u_{i}$. Es decir, en vez de considerar a $\alpha$ como fija, suponemos que es una variable aleatoria con un valor medio $\alpha$ y una desviación aleatoria $u_{i}$ de este valor medio. Sustituyendo $\alpha_{i}=\alpha+u_{i}$ en (ii) obtenemos:

$$
Y_{i t}=\alpha+\beta_{1} X_{1 i t}+u_{i}+e_{i t}
$$

Si analizamos la ecuación (iii), observamos que si la varianza de $u_{i}$ es igual a cero, es decir $\sigma_{u}^{2}=0$, entonces no existe ninguna diferencia relevante entre (i) y (iii). Para saber cuál de los dos modelos es mejor, ya sea efectos aleatorios o el de datos agrupados, aplicamos la prueba de Breusch y Pagan conocida como "Prueba del Multiplicador de Lagrange para Efectos Aleatorios". La hipótesis nula de esta prueba es que $\sigma_{u}^{2}=0$. La prueba se rechaza, si existe diferencia entre (i) y (iii), y es preferible usar el método de efectos aleatorios.

El valor "p" nos muestra que no podemos rechazar la hipótesis nula Ho, por lo que es preferible usar el método de la Regresión Agrupada (pooled M.C.o.).

Sin embrago, se requiere considerar el carácter "individual" de cada unidad de datos transversales (países); por lo tanto se procedió a especificar un modelo de Efectos Fijos para comparar cuál modelo resulta ser el óptimo.

\subsubsection{Efectos fijos (Fixed Effects)}

Otra manera de modelar el carácter "individual" de cada país es a través del modelo de efectos fijos. Este modelo no supone que las diferencias entre los 
Cuadro 2 Efectos Aleatorios (Random effects)

\begin{tabular}{|c|c|c|c|c|c|c|}
\hline \multicolumn{4}{|c|}{ Efectos Aleatorios Regresión GIS } & & Num. de Obs. & 249 \\
\hline \multicolumn{4}{|c|}{ Variable de grupo (i) : id } & & Num. de grupos & 3 \\
\hline \multirow[t]{3}{*}{ R- Cuadrada } & within & 0.6292 & & Obs.grupo & mínimo & 83 \\
\hline & between & 0.9922 & & & promedio & 83 \\
\hline & overall & 0.6462 & & & máximo & 83 \\
\hline \multicolumn{4}{|c|}{ Efectos Aleatorios U_i ～Gausiana } & Wald Chi $(4)=$ & & 445.64 \\
\hline $\operatorname{corr}\left(U_{-} l, x\right)=$ & 0 & \multicolumn{2}{|l|}{ (asumida) } & Prob>chi2= & & 0.0000 \\
\hline dlaimidl & Coef. & Error Est. & $t$ & $p>|t|$ & \multicolumn{2}{|c|}{ [Int. de Conf. 95\% ] } \\
\hline dlbccntdl & -0.718146 & 0.036607 & -19.6 & 0.000 & -0.7898938 & -0.646398 \\
\hline dlmdl & 0.2866068 & 0.03056 & 9.38 & 0.000 & 0.2267099 & 0.3465037 \\
\hline dlnwdl & 0.7441394 & 0.03783 & 19.67 & 0.000 & 0.6699938 & 0.818285 \\
\hline dltmimlrp & 0.726577 & 0.195659 & 3.71 & 0.000 & 0.343093 & 1.110061 \\
\hline cons & 318.697 & 109.4643 & 2.91 & 0.004 & 104.1509 & 533.2432 \\
\hline sigma_u & 0.0000 & & & & & \\
\hline sigma_e & 1651.6863 & & & & & \\
\hline rho & 0.0000 & & & & & \\
\hline \multicolumn{7}{|c|}{ Prueba de los multiplicadores de Lagrange de Breusch-Pagan } \\
\hline \multicolumn{7}{|c|}{ dlaimidl $[i d, t]=X b+u[i d]+e[i d, t]$} \\
\hline \multicolumn{7}{|c|}{ Resultados estimados } \\
\hline & Varianza & Des. Est. & & & & \\
\hline dlaimidl & 7734237 & 2781.05 & & & & \\
\hline e & 2728068 & 1651.686 & & & & \\
\hline$u$ & 0.0000 & 0.0000 & & & & \\
\hline \multirow[t]{3}{*}{ Prueba: } & $\operatorname{Var}(u)=$ & 0 & & & & \\
\hline & & $\operatorname{chi2}(1)$ & $=$ & 1.77 & & \\
\hline & & Prob>chi2 & $=$ & 0.1828 & & \\
\hline
\end{tabular}

Fuente: elaboración propia. 
países sean aleatorias, sino constantes o "fijas", y por ello debemos estimar cada intercepto Ui. Para que el intercepto varíe con respecto a cada país utilizamos la técnica de "las variables dicotómicas de intersección diferencial" (utilizar variables dicotómicas conduce al mismo resultado que si restamos a cada observación la media de cada país), que se expresa de la siguiente manera:

$$
Y_{i t}=v_{i}+\beta_{1} X_{1 i t}+e_{i t}
$$

Donde $v_{i}$ es un vector de variables dicotómicas para cada país.

Para saber cuál de los dos modelos es mejor, utilizamos una prueba " $F$ " restrictiva, también conocida como la prueba de Chow en la que RSS $_{R}$ es la suma de cuadrados de residuos que se obtiene de la estimación M.c.o. en el modelo agrupado y RSs $_{U}$ es la suma de cuadrados de los residuos de la estimación por mínimos cuadrados de las variables dicótomas.

Para probar la Hipótesis:

$$
\begin{aligned}
& \mathrm{Ho}=\mathrm{v}_{1}=\mathrm{v}_{2}=\ldots \ldots \ldots=\mathrm{v}_{\mathrm{i}}=0 . \\
& \mathrm{H}_{1}=\mathrm{v}_{1} \neq \mathrm{v}_{2} \neq \ldots \ldots . . \neq \mathrm{v}_{\mathrm{i}} \neq 0 .
\end{aligned}
$$

Calcular:

$$
F_{0}=\frac{\left(R S S_{R}-R S S_{U}\right) /(N-1)}{R S S_{U} /(N T-N-K)}:{ }^{H_{0}} F_{N-1, N(T-1)-K}
$$

Si F > F $F_{a}(\mathrm{~N}-1$, NT-N-K), rechazar Ho; de lo contrario, no se rechace, donde $\mathrm{F}_{\alpha}(\mathrm{N}-1)$ y $\mathrm{F}_{\alpha}(\mathrm{NT}-\mathrm{N}-\mathrm{K})$ y $(\mathrm{N}-1)$ son los grados de libertad del numerador y del denominador, respectivamente, y $\alpha$ es el nivel de significancia. Alternativamente, si el valor p-value del F obtenido de la ecuación es suficientemente bajo, se puede rechazar Ho.

En relación con el modelo (iv), el modelo (i) asume un intercepto común para todos los países (es decir, no incluye variables dicotómicas por país). Como ya se vio la hipótesis nula es que $\mathrm{v}_{1}=\mathrm{v}_{2}=\ldots=\mathrm{v}_{\mathrm{i}}=0$ (o sea, que todas las variables dicotómicas por país son iguales a cero). Si la prueba se rechaza, significa que al menos algunas variables dicotómicas sí pertenecen al modelo, y por lo tanto es necesario utilizar el método de efectos fijos.

El valor "p" nos indica que podemos rechazar la Ho, por lo que es preferible usar el método efectos fijos al modelo agrupado (pooled).

Como podemos observar, ya no es necesaria la prueba Hausman para determinar si el modelo es de efectos fijos o es de efectos aleatorios, ya que queda demostrado que el modelo óptimo es el de efectos fijos. 
Cuadro 3 Efectos Fijos (Fixed effects)

\begin{tabular}{|c|c|c|c|c|c|c|}
\hline \multicolumn{5}{|c|}{ Efectos Fijos Regresión (within) } & Num. de Obs. & 249 \\
\hline \multicolumn{5}{|c|}{ Variable de grupo (i) : id } & $\begin{array}{l}\text { Num. de } \\
\text { grupos }\end{array}$ & 3 \\
\hline \multirow{4}{*}{ R- Cuadrada } & within & 0.6296 & & Obs.grupo & mínimo & 83 \\
\hline & between & 0.9925 & & & promedio & 83 \\
\hline & overall & 0.6458 & & & máximo & 83 \\
\hline & & & & $F(4,242)=$ & & 102.82 \\
\hline $\operatorname{corr}\left(U_{-} l, x B\right)=$ & 0.2063 & & & Prob>F= & & 0.0000 \\
\hline dlaimidl & Coef. & Error Est. & $t$ & $p>|t|$ & \multicolumn{2}{|c|}{ [Int. de Conf. 95\% ] } \\
\hline dlbecntdl & -0.6990953 & 0.0370347 & -18.88 & 0.000 & -0.7720469 & -0.626144 \\
\hline dlmdl & 0.2800699 & 0.0303717 & 9.22 & 0.000 & 0.2202431 & 0.3398966 \\
\hline dlnwdl & 0.718004 & 0.0388888 & 18.46 & 0.000 & 0.6414002 & 0.7946077 \\
\hline dltmimlrp & 0.7645608 & 0.1943638 & 3.93 & 0.000 & 0.3817 & 1.147422 \\
\hline cons & 339.3755 & 108.7245 & 3.12 & 0.002 & 125.2083 & 553.5427 \\
\hline sigma_u & 347.047460 & & & & & \\
\hline sigma_e & 1651.68630 & & & & & \\
\hline rho & 0.042282 & & & & & \\
\hline \multicolumn{3}{|c|}{ Prueba $\mathrm{F}$ de que todos U_i $\mathrm{i}=0$} & $f(2,242)=$ & 3.83 & Prob $>F=$ & 0.0357 \\
\hline
\end{tabular}

Fuente: elaboración propia.

\subsubsection{Pruebas de autocorrelación y heteroscedasticidad}

Autocorrelación

Es importante señalar que aun cuando hemos modelado la heterogeneidad temporal y espacial en nuestro modelo, se debe cumplir con ciertas especificaciones. De acuerdo con los supuestos de Gauss-Markov, los estimadores de M.C.o. son los Mejores Estimadores Lineales e Insesgados (MELI) siempre y cuando los errores $\mathrm{e}_{\mathrm{it}}$ sean independientes entre sí y se distribuyan idénticamente con varianza constante $\sigma^{2}$.

Actualmente hay muchas maneras de diagnosticar problemas de autocorrelación. Wooldridge desarrolló un método en el cual se establece que la hipótesis nula de esta prueba es que no existe autocorrelación, naturalmente, si se rechaza, podemos concluir que ésta sí existe (Wooldridge, 2002). 
Eduardo Rosas Rojas

Cuadro 4 Autocorrelación (Prueba de Wooldridge)

\begin{tabular}{lc}
\hline & Prueba de Wooldridge para Autocorrelación en datos de panel \\
\hline $\mathrm{H} 0:$ & No Autocorrelación de primer orden \\
$\mathrm{F}(1,2)=$ & 8.602 \\
\hline Prob $>\mathrm{F}=$ & 0.0993 \\
\hline Fuente: elaboración propia. &
\end{tabular}

El valor "p" nos indica que No se puede rechazar la hipótesis nula Ho, por lo que no tenemos problema de Autocorrelación de primer orden.

\section{Correlación contemporánea}

Las estimaciones en datos panel pueden tener problemas de correlación contemporánea si las observaciones de ciertas unidades están correlacionadas con las observaciones de otras unidades en el mismo periodo. En la prueba de Breusch y Pagan para identificar problemas de correlación contemporánea en los residuales de un modelo de efectos fijos. La hipótesis nula es que existe "independencia transversal" (cross-sectional independence), es decir, que los errores entre las unidades son independientes entre sí.

Cuadro 5 Correlación contemporánea (Prueba xttest2)

\begin{tabular}{|c|c|c|c|}
\hline \multicolumn{4}{|c|}{ Matriz de correlaciones de los residuales } \\
\hline & el & ¿e2 & _e3 \\
\hline el & 1.0000000 & 0.0000000 & 0.0000000 \\
\hline _e2 & -0.0209000 & 1.0000000 & 0.0000000 \\
\hline _e3 & 0.0124000 & 0.0159000 & 1.0000000 \\
\hline \multirow{2}{*}{\multicolumn{2}{|c|}{ Prueba LM Breusch -Pagan: }} & $\operatorname{Chi2}(3)=$ & 2.1460 \\
\hline & & Prob.: & 0.5426 \\
\hline
\end{tabular}

Fuente: elaboración propia.

El valor "p" del estadístico $\mathrm{X}^{2}$ indica que no podemos rechazar la Ho, por lo tanto no existe problema de correlación contemporánea. 


\section{Heteroscedasticidad}

Cuando la varianza de los errores de cada unidad transversal no es constante, nos encontramos con una violación de los supuestos Gauss-Markov. Una forma de saber si nuestra estimación tiene problemas de heteroscedastidad es a través de la prueba del Multiplicador de Lagrange de Breusch y Pagan. Sin embargo, de acuerdo con Greene (2000), ésta y otras pruebas son sensibles al supuesto sobre la normalidad de los errores; afortunadamente, la prueba Modificada de Wald para Heterocedasticidad funciona aun cuando dicho supuesto es violado. La hipótesis nula de esta prueba es que no existe problema de heteroscedasticidad, es decir, $\sigma_{\mathrm{i}}^{2}=\sigma^{2}$ para toda $i=1 \ldots N$, donde $N$ es el número de unidades transversales ("países" en nuestro ejemplo). Naturalmente, cuando la Ho se rechaza, tenemos un problema de heteroscedasticidad.

Cuadro 6 Heteroscedasticidad (Prueba modificada de Wald)

Prueba Modificada de Wald de heteroscesdasticidad grupal en un modelo de efectos fijos

h0: sigma (i)^2 =para toda i

chi2 (3) $=$ 32599.09

Prob>chi2=

0.00000

Fuente: elaboración propia.

La prueba nos indica que rechazamos la Ho de varianza constante y aceptamos la Ha de Heteroscedasticidad.

\section{Corrección de Heteroscedasticidad}

El problema de heteroscedasticidad puede solucionarse con estimadores robustos, con Mínimos Cuadrados Generalizados Factibles (FGLS), o bien con Errores Estándar Corregidos para Panel (PCSE). Beck y Katz (2001) demostraron que los errores estándar de PCSE son más precisos que los de FGLS. Desde entonces muchos trabajos en la disciplina han utilizado PCSE en sus estimaciones para datos de panel. 
Eduardo Rosas Rojas

Tabla 7 Errores estándar corregidos para panel

\begin{tabular}{|c|c|c|c|c|c|c|}
\hline \multicolumn{4}{|c|}{ Variable de grupo: id } & & \multirow{2}{*}{$\begin{array}{l}\text { Num. de Obs. } \\
\text { Num. de } \\
\text { grupos }\end{array}$} & 249 \\
\hline \multicolumn{2}{|l|}{ Variables de tiempo } & \multicolumn{3}{|l|}{ periodo } & & 3 \\
\hline Panel: & \multicolumn{2}{|c|}{ heteroscedasticidad } & \multicolumn{2}{|c|}{ (balanceada) } & Obs. Por grupo & \\
\hline \multirow[t]{3}{*}{ Autocorrelación: } & within & \multirow{3}{*}{\multicolumn{2}{|c|}{ № Autocorrelación }} & \multirow[t]{3}{*}{ Obs.grupo } & mínimo & 83 \\
\hline & & & & & promedio & 83 \\
\hline & & & & & máximo & 83 \\
\hline \multicolumn{2}{|c|}{ Covarianzas estimadas } & \multicolumn{2}{|l|}{3} & \multicolumn{2}{|l|}{ R.cuadrada } & 0.6962 \\
\hline \multicolumn{2}{|c|}{ Autocorrelaciones estimadas } & \multicolumn{2}{|l|}{0} & \multicolumn{2}{|c|}{ Wald chi2 (4) } & 258.5000 \\
\hline \multicolumn{2}{|c|}{ Coeficientes estimados } & \multicolumn{2}{|l|}{5} & \multicolumn{2}{|l|}{ Prob>chi2 } & 0.00000 \\
\hline \multicolumn{7}{|c|}{ HETEROSCEDASTICIDAD CORREGIDA } \\
\hline dlaimidl & Coef. & Error Est. & Z & $p>|Z|$ & \multicolumn{2}{|c|}{ [Int. de Conf. 95\% ] } \\
\hline dlbccntdl & -0.71815 & 0.0482039 & -14.90 & 0.000 & -0.8126238 & -0.6236681 \\
\hline dlmdl & 0.28661 & 0.0439751 & 6.52 & 0.000 & 0.2004172 & 0.3727965 \\
\hline dlnwdl & 0.74414 & 0.0512651 & 14.52 & 0.000 & 0.6436617 & 0.8446171 \\
\hline dltmimlrp & 0.72658 & 0.2204008 & 3.30 & 0.001 & 0.2945993 & 1.158555 \\
\hline cons & 318.6970 & 104.3905 & 3.05 & 0.002 & 114.0953 & 523.2987 \\
\hline
\end{tabular}

Fuente: elaboración propia.

Podemos ver que los coeficientes tienen los signos esperados además de que son estadísticamente significativos. Lo anterior nos muestra el modelo de acumulación de reservas óptimo para datos de panel, el valor "p" de la variable constante sigue teniendo una t poco significativa aunque mejoró con respecto a la regresión de efectos fijos. 


\section{BIBLIOGRAFÍA}

Ball, L., "Policy Rules for Open Economies", en J. B. Taylor (Ed.), Monetary Policy Rules, University of Chicago Press, 1999.

Beck, Nathaniel and Jonathan Katz. 2001. "Random Coefficient Models for Time-Series-Cross-section Data." Presented at the 2001 Annual Meeting of the Society for Political Methodology, Emory University.

Böfinger P., Monetary Policy: Goals, Institutions, Strategies and Instruments, Oxford University Press, 2001.

Böfinger P. y T. Wollmershäuser, "Managed Floating: Understanding the New International Monetary Order", Centre for Economic Policy Research (CEPR), Discussion Paper num. 3064, November, 2001.

Burdisso, Tamara. "Estimación de una función de costos para los bancos privados argentinos utilizando datos de panel, Banco Central de la República Argentina”, Documentos de Trabajo núm. 3, 1997.

Calvo, G. A. y C. M. Reinhart, "Fear of Floating", Quarterly Journal of Economics, Vol. 117, num. 2, May, 2002.

Canales-Kriljenko, J. I., "Foreign Exchange Intervention in Developing and Transition Economies: Results of a Survey", IMF Working Paper WP/03/95, May, 2003.

Dickey, D., W. Fuller, Distribution of the Estimators for Autoregressive Time Series with a Unit Root, Journal of the American Statistical Association 74, 1979, pp. 427-31.

Flood, R. P. y A. K. Rose, "Uncovered Interest Parity in Crisis: the Interest Rate Defence in the 1990s, Centre for Economic Policy Research (CEPR), Discussion Paper num. 2943, 2001.

Frenkel, R., "La sostenibilidad de la política de esterilización", Centro de Economía y Finanzas para el Desarrollo de la Argentina (CEFIDAR), Documento de Trabajo núm. 17, agosto, 2007.

Frenkel, R., "Tipo de cambio real competitivo, inflación y política monetaria” Revista de la Cepal 96, diciembre 2008.

Granger, C.W.J., P. Newbold, "Spurious Regressions in Econometrics", Journal of Econometrics 2, 1974, pp.111-120.

Greene, W.. Econometric Analysis, Upper Saddle River, nJ, Prentice Hall, 2000, p. 598.

Hernández Barriga, P., "Tipos de cambio e inflación en América Latina”, en G. Mántey y Teresa S. López (coords.), Política monetaria con elevado traspaso del tipo de cambio. La experiencia mexicana con metas de inflación, México, unAM-Plaza y Valdés, 2009. 
Hsiao, Cheng, Analysis of Panel Data, Econometric Society Monographs. Cambridge University Press, 1986.

Hüfner F., "Foreign Exchange Intervention as a Monetary Policy Instrument: Evidence for Inflation Targeting Countries", zew Economic Studies 23, Centre for European Economic Research, Physica-Verlag, Heidelberg, 2004.

Ibarra Carlos, "Is Latin America Overcoming its Fear of Floating?", Cuadernos de Economía, Vol. 44, noviembre, 2007, pp. 183-209.

Im, K., Pesaran, M. H., Shin, Y., Testing for Unit Roots in Heterogeneous Panels, Journal of Econometrics 115, 2003. pp. 53-74.

Kalecki, M., Theory of Economic Dynamics (Teoría de la dinámica económica, Fondo de Cultura Económica, México, 1985), 1954.

Levin, A., Lin, C. F., "Unit Root Tests in Panel Data: Asymptotic and Finite Sample Properties", Discussion Paper num. 92-93, University of California at San Diego, 1992.

Li, J. y R. S. Rajan , "Can High Reserves Offset Weak Fundamentals? A Simple Model of Precautionary Demand for Reserves", Lee Kuan Yew School of Public Policy Working Paper 13-05, 2005.

Mántey de Anguiano, G., Lecciones de economía monetaria, unam, México, 1997, p. 112.

Mántey de Anguiano, G., Intervención esterilizada en el mercado de cambios en un régimen de metas de inflación: la experiencia de México, Investigación económica, vol. LXVIII, número especial 2009, pp. 47-78.

Reinhart, C. M. y K. S. Rogoff, "The Modern History of Exchange Rate Arrangements: a Reinterpretation", Quarterly Journal of Economics, Vol. CxIx, num. 1, February, 2004.

Romer, D., "Keynesian Macroeconomics Without the LM Curve", Journal of Economic Perspectives, Vol. 14, num. 2, Spring, 2000.

Sarno, Lucio and Taylor, Mark P., "Official Intervention in the Foreign Exchange Market”, Unpublished Manuscript, University College, Oxford, 2000.

Svensson, L. E. O., "Open Economy Inflation Targeting”, Journal of International Economics, num. 50, 2000.

Toporowski, J., Limitaciones a la estabilización financiera por los bancos centrales, en G. Mántey y N. Levy (Coords.), Inflación, crédito y salarios: nuevos enfoques de política monetaria para mercados imperfectos, Cámara de DiputadosUNAM-M. A. Porrúa, México, 2005.

Wooldridge, J. M., Econometric Analysis of Cross Section and Panel Data, Cambridge, MA: MIT Press, 2002. 